\title{
Two-dimensional simulations of mixing in classical novae: The effect of white dwarf composition and mass ${ }^{\star}$
}

\author{
Jordi Casanova ${ }^{1}$, Jordi Josée ${ }^{2,3}$, and Steven N. Shore ${ }^{4}$ \\ 1 Physics Division, Oak Ridge National Laboratory, PO Box 2008, Oak Ridge, TN 37831-6354, USA \\ e-mail: novaj@ornl.gov \\ 2 Departament de Física, EEBE, Universitat Politècnica de Catalunya, c/Eduard Maristany 10, 08930 Barcelona, Spain \\ 3 Institut d'Estudis Espacials de Catalunya, c/Gran Capità 2-4, Ed. Nexus-201, 08034 Barcelona, Spain \\ 4 Dipartimento di Física "Enrico Fermi”, Università di Pisa and INFN, Sezione di Pisa, Largo B. Pontecorvo 3, 56127 Pisa, Italy
}

Received 14 May 2018 / Accepted 25 July 2018

\begin{abstract}
Context. Classical novae are explosive phenomena that take place in stellar binary systems. They are powered by mass transfer from a low-mass main sequence star onto either a $\mathrm{CO}$ or ONe white dwarf. The material accumulates for $10^{4}-10^{5} \mathrm{yr}$ until ignition under degenerate conditions, resulting in a thermonuclear runaway. The nuclear energy released produces peak temperatures of $\sim 0.1-0.4 \mathrm{GK}$. During these events, $10^{-7}-10^{-3} M_{\odot}$ enriched in intermediate-mass elements, with respect to solar abundances, are ejected into the interstellar medium. However, the origin of the large metallicity enhancements and the inhomogeneous distribution of chemical species observed in high-resolution spectra of ejected nova shells is not fully understood.

Aims. Recent multidimensional simulations have demonstrated that Kelvin-Helmholtz instabilities that operate at the core-envelope interface can naturally produce self-enrichment of the accreted envelope with material from the underlying white dwarf at levels that agree with observations. However, such multidimensional simulations have been performed for a small number of cases and much of the parameter space remains unexplored.

Methods. We investigated the dredge-up, driven by Kelvin-Helmholtz instabilities, for white dwarf masses in the range $0.8-1.25 M_{\odot}$ and different core compositions, that is, $\mathrm{CO}$-rich and ONe-rich substrates. We present a set of five numerical simulations performed in two dimensions aimed at analyzing the possible impact of the white dwarf mass, and composition, on the metallicity enhancement and explosion characteristics.

Results. At the time we stop the simulations, we observe greater mixing ( $\sim 30 \%$ higher when measured in the same conditions) and more energetic outbursts for ONe-rich substrates than for CO-rich substrates and more massive white dwarfs.
\end{abstract}

Key words. novae, cataclysmic variables - nuclear reactions, nucleosynthesis, abundances - hydrodynamics - instabilities convection - turbulence

\section{Introduction}

Classical novae are stellar explosions powered by thermonuclear runaways (TNR) on the surface of a white dwarf after an accretion episode of hydrogen-rich matter from the companion star at typical rates of $\sim 10^{-10}-10^{-9} M_{\odot} \mathrm{yr}^{-1}$. The TNR drives peak temperatures of $\sim(1-4) \times 10^{8} \mathrm{~K}$. Between $10^{-7}$ and $10^{-3} M_{\odot}$ are ejected with peak velocities that achieve several thousand $\mathrm{km} \mathrm{s}^{-1}$. The suite of nuclear processes that operate in the envelope results in nonsolar isotopic abundance ratios in the ejecta (Gehrz et al. 1998; Downen et al. 2013; Kelly et al. 2013). Classical novae are spectroscopically classified as neon novae and non-neon novae. Neon novae are characterized by the presence of intense Ne lines and are thought to take place on $\mathrm{ONe}$ white dwarfs; non-neon novae are absent of $\mathrm{Ne}$ lines and take place on $\mathrm{CO}$ white dwarfs. Novae contribute to the production of Galactic ${ }^{15} \mathrm{~N},{ }^{17} \mathrm{O}$, and ${ }^{13} \mathrm{C}$ (Starrfield et al. 2008, 2016; José \& Hernanz 1998; José 2016). Other species, such as ${ }^{31} \mathrm{P},{ }^{32} \mathrm{~S}$, and ${ }^{35} \mathrm{Cl}$ can also be produced in the most massive ONe novae, since the pressure achieved at the base of the accreted envelope is higher in these novae. In ONe novae, this translates into higher peak temperatures and

\footnotetext{
^ The movies associated to Fig. 1 are available at https://www . aanda.org
}

nuclear processing that extends toward heavier isotopes, beyond the CNO region (José 2016; José \& Shore 2008).

While the matter accreted from the companion onto the white dwarf has approximately solar composition $(Z \sim 0.02)$, the ejecta does not. The metallicity enhancements inferred from observations reveal values in a wide range from slightly above solar to $Z \sim 0.80^{1}$ for the more massive ONe novae (see Vanlandingham et al. 1997 and Gehrz et al. 1998, and references therein). Owing to the moderately low peak temperatures achieved during the outburst, nuclear processing in the accreted envelope alone is unlikely to account for the high metallicities inferred from observations. Instead, mixing at the core-envelope interface (CEI) is a more reliable alternative to explain the metallicity enhancement in the ejecta. One-dimensional, spherical symmetric models often assume mixing, at a constant rate, between the material transferred from the companion and the outermost layers of the underlying white dwarf, whose composition reflects its previous evolutionary history. Such mixing is, however, most likely time dependent. For the 1D simulations, several mixing mechanisms have been proposed, such as diffusive

\footnotetext{
1 For example, V1370 Aql 1982 (Snijders et al. 1987; Andreä et al. 1994), although it is worth mentioning that these studies used ionization correction methods.
} 
mixing (Prialnik \& Kovetz 1984; Kovetz \& Prialnik 1985; Fujimoto \& Iben 1992; Iben et al. 1991, 1992) and shear mixing (Durisen 1977; Kippenhahn \& Thomas 1978; MacDonald 1983; Livio \& Truran 1987; Fujimoto 1988; Sparks \& Kutter 1987; Kutter \& Sparks 1987, 1989). Yet none can reproduce the full range of metallicity enhancements inferred from observations (Livio \& Truran 1990). Aside from the difficulties faced by 1D models in the search for a feasible mixing mechanism, spherically symmetric simulations cannot explore how the ignition begins and how the deflagration spreads throughout the accreted envelope (Shara 1982). Therefore, multidimensional hydrodynamical calculations are required to shed more light on the unexplained features of classical nova explosions, in particular, the role of fluid instabilities for the CNO enhancement.

Recent studies in 2D and 3D simulations (see Glasner et al. 2012; Casanova et al. 2016, and references therein) confirmed that Kelvin-Helmholtz instabilities at the CEI can dredge up material from the underlying white dwarf and efficiently enrich the accreted envelope to levels in agreement with observations. While most of the multidimensional nova studies performed to date have focused on mixing with $\mathrm{CO}$-rich substrates, two independent efforts have analyzed the effect of various compositions on the underlying white dwarf (Glasner et al. 2012, 2014; Casanova et al. 2016). The 2D models by Glasner et al. $(2012,2014)$ assumed mass-accretion of solar composition onto a $1.147 M_{\odot}$ white dwarf with a maximum resolution of $1.4 \times$ $1.4 \mathrm{~km}^{2}$ in spherical-polar coordinates. The authors found that Kelvin-Helmholtz instabilities can enrich the accreted envelope with material from the underlying white dwarf, independent of the nature of the chemical substrate $\left(\mathrm{CO}\right.$, pure He, pure ${ }^{16} \mathrm{O}$, and pure ${ }^{24} \mathrm{Mg}$ ), but the study did not consider a realistic composition of an ONe white dwarf. The 3D work in Cartesian coordinates by Casanova et al. (2016) employed instead an ONe-rich substrate model based on Ritossa et al. (1996), assuming accretion of solar composition onto a $1.25 M_{\odot}$ ONe white dwarf with a maximum resolution of $1.56 \times 1.56 \times 1.56 \mathrm{~km}^{3}$. The authors found larger metallicity enhancements and longer durations of TNR for ONe-rich substrates, but further analysis of the influence of the core composition for various white dwarf masses is needed. In this paper, we present a 2D study of mixing at the CEI during nova outbursts with self-consistent models to explore the effect of the white dwarf mass and composition and with the aim of understanding the impact of white dwarf gravity for a given chemical composition.

\section{Input physics and initial setup}

We used the 1D implicit Lagrangian hydrodynamic code SHIVA to simulate the accretion of solar composition material $(Z=$ 0.02 ) onto the white dwarf at a rate of $2 \times 10^{-10} M_{\odot} \mathrm{yr}^{-1}$ (José \& Hernanz 1998; José 2016). We assumed cold accretion with no premixing, at a constant rate. For this study, we computed three models of CO-rich and two models of ONe-rich substrates with different white dwarf masses: $0.8 M_{\odot} \mathrm{CO}, 1.0 M_{\odot}$ $\mathrm{CO}, 1.15 M_{\odot} \mathrm{CO}, 1.15 M_{\odot} \mathrm{ONe}$, and 1.25 $M_{\odot} \mathrm{ONe}$. For CO-rich substrate models, the composition of the underlying white dwarf is $\mathrm{X}\left({ }^{12} \mathrm{C}\right)=\mathrm{X}\left({ }^{16} \mathrm{O}\right)=0.5$. For ONe-rich substrate models, we adopted the composition of the outer white dwarf layers from Ritossa et al. (1996): $\mathrm{X}\left({ }^{16} \mathrm{O}\right)=0.511, \mathrm{X}\left({ }^{20} \mathrm{Ne}\right)=0.313, \mathrm{X}\left({ }^{23} \mathrm{Na}\right)$ $=0.0644, \mathrm{X}\left({ }^{24} \mathrm{Mg}\right)=0.0548, \mathrm{X}\left({ }^{25} \mathrm{Mg}\right)=0.0158, \mathrm{X}\left({ }^{27} \mathrm{Al}\right)=$ $0.0108, \mathrm{X}\left({ }^{12} \mathrm{C}\right)=0.00916, \mathrm{X}\left({ }^{26} \mathrm{Mg}\right)=0.00989, \mathrm{X}\left({ }^{21} \mathrm{Ne}\right)=$ 0.00598 , and $\mathrm{X}\left({ }^{22} \mathrm{Ne}\right)=0.00431$.

The structures were mapped onto a 2D Cartesian grid when the CEI temperature reached $T=0.1 \mathrm{GK}$, and the subsequent evolution was followed with the multidimensional parallelized explicit Eulerian FLASH code. The FLASH code is based on the piecewise parabolic interpolation of physical quantities to solve the hydrodynamic equations that describe the stellar plasma. This code has a timestep limited by the Courant-FriedrichsLewy condition and uses adaptive mesh refinement to resolve critical features along the computational domain (Fryxell et al. 2000). Thermal diffusion is implemented by adding the heat flux in the energy equation, which is calculated using a Rossland mean opacity that includes radiative and conductive contributions (Timmes 2000). The physical description of the fluid is closed by the Helmholtz equation of state, which is suitable for degenerate stellar matter and includes contributions from ions treated as an ideal gas, radiation as a blackbody, and degenerate electrons and positrons described with a noninteracting Fermi gas (Timmes \& Arnett 2000; Timmes \& Swesty 2000). The FLASH code uses dissipation algorithms, as described in Colella \& Woodward (1984), such as a flattening procedure and a monotonicity constraint (rather than artificial viscosity) to control oscillations near discontinuities; this feature is shared with the MUSCL scheme of van Leer (van Leer 1979). The calculations were performed on the MareNostrum supercomputer (Barcelona Supercomputing Center).

The 2D computational domain for the $\mathrm{CO}$ nova models is $800 \times 800 \mathrm{~km}^{2}$, initially comprising 128 unevenly spaced radial layers, and 1024 equally spaced grid points along the horizontal axis. For the $\mathrm{ONe}$ nova models, the $2 \mathrm{D}$ computational domain is $800 \times 400 \mathrm{~km}^{2}$ and has 96 unevenly spaced radial zones and 1024 equally spaced zones along the horizontal axis. The initial spacing is determined by the refinement criterion used by the FLASH code, which tends to refine the regions characterized by rapidly changing physical variables (i.e., the CEI rather than other regions of the accreted envelope). The critical mass and extent of the accreted envelopes depend sensitively on the properties of the underlying white dwarf. The envelope is more massive and larger for CO-rich than for the more massive ONe-rich white dwarfs, although the latter reach higher densities because of the white dwarf mass-radius relation (Starrfield et al. 1998; José \& Hernanz 1998; Yaron et al. 2005; José 2016). A maximum resolution of $0.78 \times 0.78 \mathrm{~km}^{2}$ was adopted to capture the mixing process operating at the CEI in all the simulations reported in this paper. Initially, the structure was forced to be in hydrostatic equilibrium throughout the envelope. This condition is reinforced with a reflecting boundary condition at the bottom (i.e., the sign of the velocity is reversed), and an outflow condition at the top, which allows matter to escape. Periodic lateral boundary conditions were adopted (Zingale et al. 2002; Casanova et al. 2010, 2011a,b, 2016).

We employed a reduced nuclear network containing 13 chemical species $\left({ }^{1} \mathrm{H},{ }^{4} \mathrm{He},{ }^{12,13} \mathrm{C},{ }^{13,14,15} \mathrm{~N},{ }^{14,15,16,17} \mathrm{O}\right.$, and ${ }^{17,18} \mathrm{~F}$ ) linked through 18 nuclear reactions, mainly p-captures and $\beta^{+}$-disintegrations, to compute the energetics of $\mathrm{CO}$-rich novae (Casanova et al. 2010, 2011a,b). For ONe-rich novae, we employed an extended nuclear network containing 31 chemical species $\left({ }^{1} \mathrm{H},{ }^{4} \mathrm{He},{ }^{12,13} \mathrm{C},{ }^{13,14,15} \mathrm{~N},{ }^{14,15,16,17} \mathrm{O},{ }^{17,18} \mathrm{~F},{ }^{20,21} \mathrm{Ne}\right.$, ${ }_{21,22,23} \mathrm{Na},{ }^{22,23,24,25,26} \mathrm{Mg},{ }^{24,25,26 \mathrm{~g}, 26 \mathrm{~m}, 27} \mathrm{Al}$, and ${ }^{26,27,28} \mathrm{Si}$ ), since the main nuclear processing extends beyond the CNO cycle in this type of novae (Casanova et al. 2016). The reaction rates were taken from STARLIB nuclear reaction library (Iliadis et al. 2010).

\section{Results}

We initiated the simulations by introducing a top-hat temperature perturbation $(5 \%)$ operating only at the first timestep in 
a $1 \mathrm{~km}$ wide region located near the CEI. The location of the perturbation is the same for the five computed models. Previous work (Casanova et al. 2011a) has shown that the outcome is insensitive to the nature of the perturbation. The perturbation disrupts the initial equilibrium configuration and creates fluctuations at the interface that spawn strong buoyant fingers. In Fig. 1, panel a shows the development of these primary fluctuations at $t=54 \mathrm{~s}$ for the $1.0 M_{\odot} \mathrm{CO}$ model. Panels b-f in Fig. 1 show the progress of the convective front at the time it is located at 4 pressure scale heights above the CEI, for the different models reported in this work. The scale pressure height, at the times indicated in Fig. 1, is calculated as $H_{\mathrm{p}}=k T / m g$, where $k$ is the Boltzmann constant, $T$ is the temperature, $m$ is the molecular mass, and $g$ is the value of the gravity. The two $1.15 M_{\odot}$ models have the same gravity, but once mixing operates, the values of $T$ and $m$ differ, resulting in different values of $H_{\mathrm{p}}$. Four pressure scale heights correspond to $627.2 \mathrm{~km}, 284.6 \mathrm{~km}, 146.9 \mathrm{~km}, 110.5 \mathrm{~km}$, and $66.4 \mathrm{~km}$ above the CEI for models $0.8 M_{\odot} \mathrm{CO}, 1.0 M_{\odot} \mathrm{CO}, 1.15 M_{\odot}$ $\mathrm{CO}, 1.15 M_{\odot} \mathrm{ONe}$, and $1.25 M_{\odot} \mathrm{ONe}$, respectively. Shear flows develop at the interface and trigger strong Kelvin-Helmholtz instabilities that efficiently dredge-up core material and enrich the envelope in metals. The convective front progresses subsonically upward almost uniformly, such that the event proceeds almost in spherical symmetry. That explains the success of 1D calculations in describing the main characteristics of classical nova explosions (Starrfield et al. 1998; Kovetz \& Prialnik 1997; Yaron et al. 2005; José \& Hernanz 1998).

The advance of the convective front is limited by the abundance stratification and temperature gradient within the accreted envelope. Consequently, matter burns layer by layer depending on the composition and temperature that characterizes each stratum, and the front moves upward almost uniformly. Figure 2 demonstrates the advance of the convective front as a function of time until the convective front has nearly reached the upper boundary at $t=310 \mathrm{~s}$ and $t=657 \mathrm{~s}$ for models $1.0 M_{\odot}$ $\mathrm{CO}$ and $1.25 M_{\odot} \mathrm{ONe}$, respectively. As burning proceeds, convection becomes more disordered ${ }^{2}$ and the $2 \mathrm{D}$ convective cells continue growing in size, occupying almost the entire domain length. As opposed to the 3D case (Casanova et al. 2011b, 2016), conservation of vorticity in 2D forces eddies to grow in an inverse vorticity cascade with a distribution that deviates from the Kolmogorov spectrum (Pope 2000; Lesieur 2008; Shore 2007). In a $3 \mathrm{D}$ framework, the fluid shows a more chaotic pattern, where filaments originating at the CEI tend to break into smaller structures while being engulfed at the upper parts of the envelope. The formation of long-lived eddies of multiple sizes is a signature of the intermittent behavior of turbulence. Movies showing the development of the Kelvin-Helmoltz instabilities and the progress of the convective front for models $1.0 M_{\odot}$ $\mathrm{CO}$ and $1.25 M_{\odot} \mathrm{ONe}$ are available online ${ }^{3}$. We note that the movie of the $1.25 M_{\odot}$ ONe shows a Landau-like bifurcation at $\sim 100$ s (Landau \& Lifshitz 1987). This bifurcation appears when nonlinear modes become strong enough that the fluid becomes unstable and develops an oscillatory transition into a more tangled regime. In Fig. 3, we show the total velocity histograms at the end of the simulation for model $1.0 M_{\odot} \mathrm{CO}$ at $t=$ $310 \mathrm{~s}\left(\right.$ mean velocity $=1.06 \times 10^{7} \mathrm{~cm} \mathrm{~s}^{-1}$ and dispersion $=6.74 \times$

\footnotetext{
2 Based on the prescription by Spitzer (1962) as implemented in the FLASH code, typical values of the Reynolds and Prandtl numbers are $\sim 10^{19}$ and $\sim 10^{-7}$, respectively.

3 Or at http://www. fen.upc.edu/users/jjose/Downloads. html
}

$10^{6} \mathrm{~cm} \mathrm{~s}^{-1}$ ), and model $1.25 M_{\odot} \mathrm{ONe}$ at $t=657 \mathrm{~s}$ (mean velocity $=1.87 \times 10^{6} \mathrm{~cm} \mathrm{~s}^{-1}$ and dispersion $\left.=1.48 \times 10^{6} \mathrm{~cm} \mathrm{~s}^{-1}\right)$. Model 1.0 $M_{\odot} \mathrm{CO}$ presents a more developed turbulent regime that translates into a distribution that is approximately Gaussian with higher mean velocity and a lower dispersion. The $1.25 M_{\odot}$ ONe model instead presents a distribution with a larger dispersion and lower velocities, indicating that turbulence is less developed. The same trend is found in the cumulant distribution functions of the Mach number for these models (see Fig. 4). Mean values of the Mach numbers and their corresponding variances are $\bar{M} a=0.0862$ and $\sigma^{2}=0.0002$ for model $1.0 M_{\odot} \mathrm{CO}$, and $\bar{M} a=0.0180$ and $\sigma^{2}=0.0024$ for model $1.25 M_{\odot}$ ONe. The inhomogenous distribution with an extended tail is distinctly nonGaussian, indicating that these quantities present a signature of the intermittent behavior of the turbulence (Casanova et al. 2011b).

When the convective front reached 4 pressure scale heights above the interface, we obtain reference parameters for the five computed models. In Table 1, we list the main characteristics at this time: white dwarf substrate (Nova), size of the computational domain (Size), mass of the white dwarf $\left(M_{\mathrm{wd}}\right)$, the value of the gravity at the CEI $(g)$, rise time of the initial buoyant fingering $\left(t_{\text {inst }}\right)^{4}$, value of the pressure scale height $(\mathrm{Hp})$, distance above the CEI at which the convective front is at $(d)$, final computed time at which the convective front is at 4 pressure scale heights above the CEI $\left(t_{\mathrm{Hp}}\right)$, mean metallicity throughout the accreted envelope $(Z)$, and base temperature $\left(T_{\text {base }}\right)$. The initial temperature perturbation diffuses rapidly and creates hot spots that burn faster and transfer energy to the surrounding material, which becomes buoyant. The initial buoyant fingering appears sooner in the simulations for ONe-rich substrates and for more massive white dwarfs owing to higher density values in the accreted envelope and steeper density gradients which, in turn, translate into a higher pressure at the base (see $t_{\text {inst }}$ in Table 1, and panel a in Fig. 5; José 2016). The buoyant fingering time for the ONe models and the formation of small convective eddies in the innermost layers of the envelope is delayed relative to the $\mathrm{CO}$ models. For instance, in the $1.25 M_{\odot}$ ONe model, it takes $\sim 35 \mathrm{~s}$ for the initial buoyant fingering to develop convection, while it only takes $\sim 20 \mathrm{~s}$ in the $1.0 M_{\odot} \mathrm{CO}$ model (see movies). Rising plumes contain hot gas that is replaced by material from the cold upper layers and effective circulation initiates at the innermost layers of the accreted envelope. However, when this occurs depends on the ignition timescale. For solar material ${ }^{5}$, ignition is driven by the reaction ${ }^{12} \mathrm{C}+\mathrm{p}$, which is faster than other channels, such as ${ }^{16} \mathrm{O}+\mathrm{p}$ or ${ }^{20} \mathrm{Ne}+\mathrm{p}$ (José et al. 2016). Hence, ignition and the establishment of superadiabatic gradients required for convection occur earlier in CO-rich substrates ${ }^{6}$. In ONe-rich substrates, the amount of ${ }^{12} \mathrm{C}$ that can be excavated from the core by Kelvin-Helmholtz instabilities is small compared to CO-rich substrates $\left(\mathrm{X}\left(\mathrm{C}^{12}\right)=0.00916\right.$ and $\mathrm{X}\left(\mathrm{C}^{12}\right)=0.5$, respectively), which results in a longer dredge-up episode, and therefore, a higher degree of mixing (Casanova et al. 2016). The timescale of the explosion, as well as the completeness of convection, is consequently longer in ONe-rich substrates, requiring more than $600 \mathrm{~s}$ to reach the upper boundary, as opposed to $\sim 300 \mathrm{~s}$ in CO-rich substrates (see movies) ${ }^{7}$. We also note that

$4 t_{\text {inst }}$ corresponds to the time at which the first instability arises at the CEI for each computed model.

5 See, however, Shen \& Bildsten (2009) for ignition conditions in C-poor envelopes.

6 We note that superadiabatic gradients are not a requirement for buoyancy.

7 It is worth mentioning that the extent of the accreted envelope is twice as large as those of the ONe-rich substrate models. 

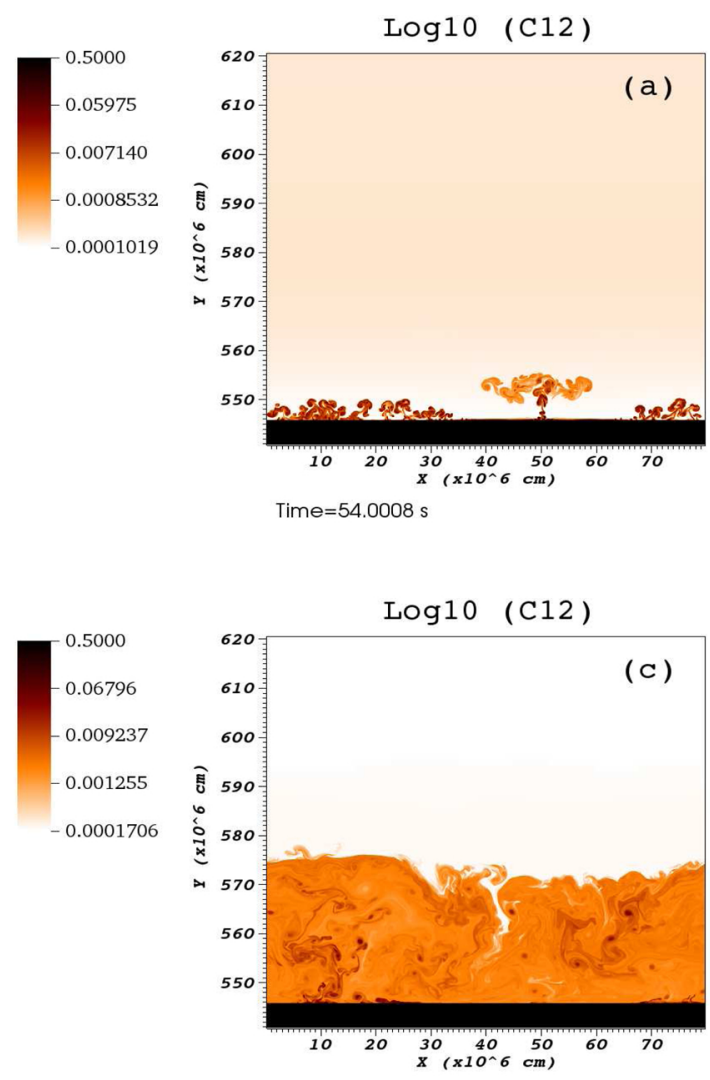

Time $=149.001 \mathrm{~s}$

$\log 10(\mathrm{Ne} 20)$
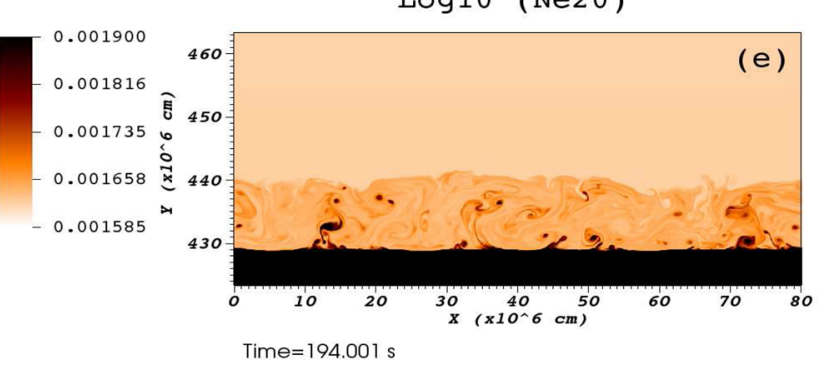
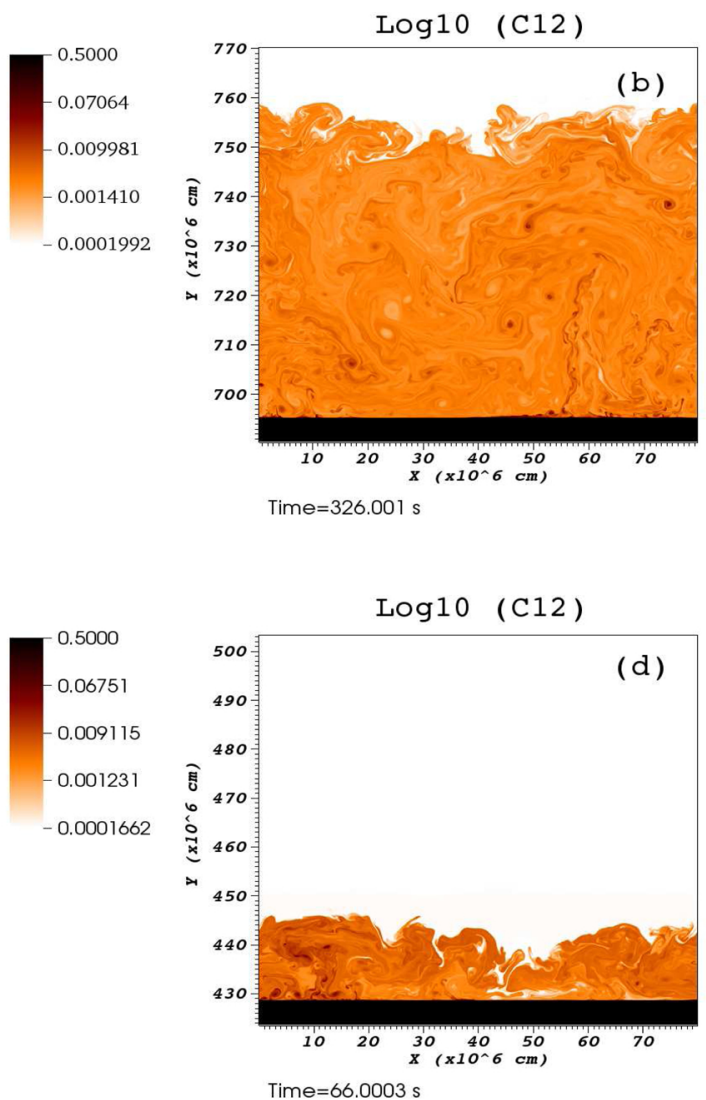

$\log 10(\mathrm{Ne} 20)$
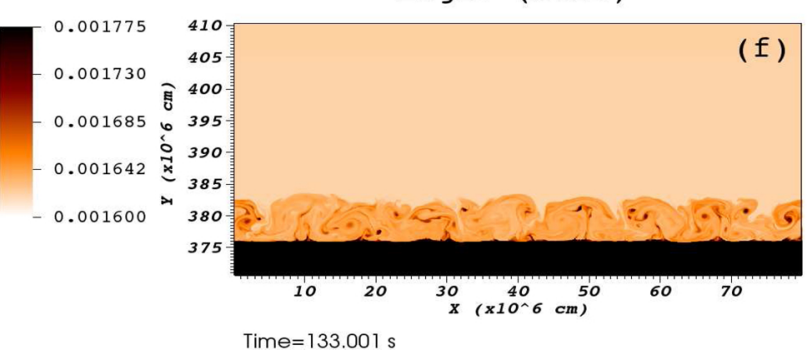

Fig. 1. Panel $a$ shows the growth of the primary fluid instabilities at $t=54 \mathrm{~s}$, in terms of ${ }^{12} \mathrm{C}$ mass fraction and in logarithmic scale, for model $1.0 M_{\odot} \mathrm{CO}$ white dwarf. The other panels show snapshots of the convective front when it is at a distance of 4 pressure scale heights above the CEI for models $0.8 M_{\odot} \mathrm{CO}($ panel $b), 1.0 M_{\odot} \mathrm{CO}$ (panel c), $1.15 M_{\odot} \mathrm{CO}$ (panel d), $1.15 M_{\odot} \mathrm{ONe}$ (panel e), and $1.25 M_{\odot} \mathrm{ONe}($ panel $f$ ). The snapshots are shown in terms of ${ }^{12} \mathrm{C}$ mass fraction for CO-rich substrates, and in terms of ${ }^{20} \mathrm{Ne}$ mass fraction for ONe-rich substrates, both in logarithmic scale. The times are $t=326 \mathrm{~s}, t=149 \mathrm{~s}, t=66 \mathrm{~s}, t=194 \mathrm{~s}$, and $t=133 \mathrm{~s}$, respectively. The reflection condition is imposed in the lower boundary, which is located $50 \mathrm{~km}$ below the CEI for all the simulations. See movies available online.

the convective front propagates faster in $\mathrm{CO}$-rich substrates (see Fig. 2). In Fig. 5, panel b shows a comparison of the evolution of the metallicity between models $1.15 M_{\odot} \mathrm{CO}$ and $1.15 M_{\odot}$ $\mathrm{ONe}$ at the time the convective front reached 4 pressure scale heights. The mean metallicities are indeed higher at the time we stop the simulations, $\sim 30 \%$, in ONe-rich substrates $(Z=$ $0.0330 \pm 0.0045$ in CO-rich substrates versus $Z=0.0530 \pm 0.0003$ in ONe-rich substrates). We observe a much more homogeneous metallicity enhancement for ONe models; we note the smaller dispersion that these models present in panel b of Fig. 5. Mixing is less uniform in CO-rich substrates because a larger amount of ${ }^{12} \mathrm{C}$ can be dredged up from the core. Therefore, burning proceeds faster, resulting in a more developed turbulent regime, which in turn, translates into a more inhomogeneous filamentary behavior. The trend observed in the final metallic- ities is also found in spherical (1D) models José \& Hernanz 1998.

It is worth mentioning that the burning front for these models is the boundary of the convecting region. Its advance is the consequence of the time development of the nuclear source and the nonlinear process of thermally buoyant motions inducing dynamical instabilities. We cannot capture the fully developed stage because of the relatively short duration of the simulations, relatively limited size of the computational domain, and restriction of all motions to two dimensions. But notwithstanding these limitations the models suffice to make some firm statements. There is a cascade, to the extent that a broad spectrum of motion and density clearly develops, such that the models lead to a broad hierarchy of mixing with large coherent structures persisting that differ in chemical composition. 

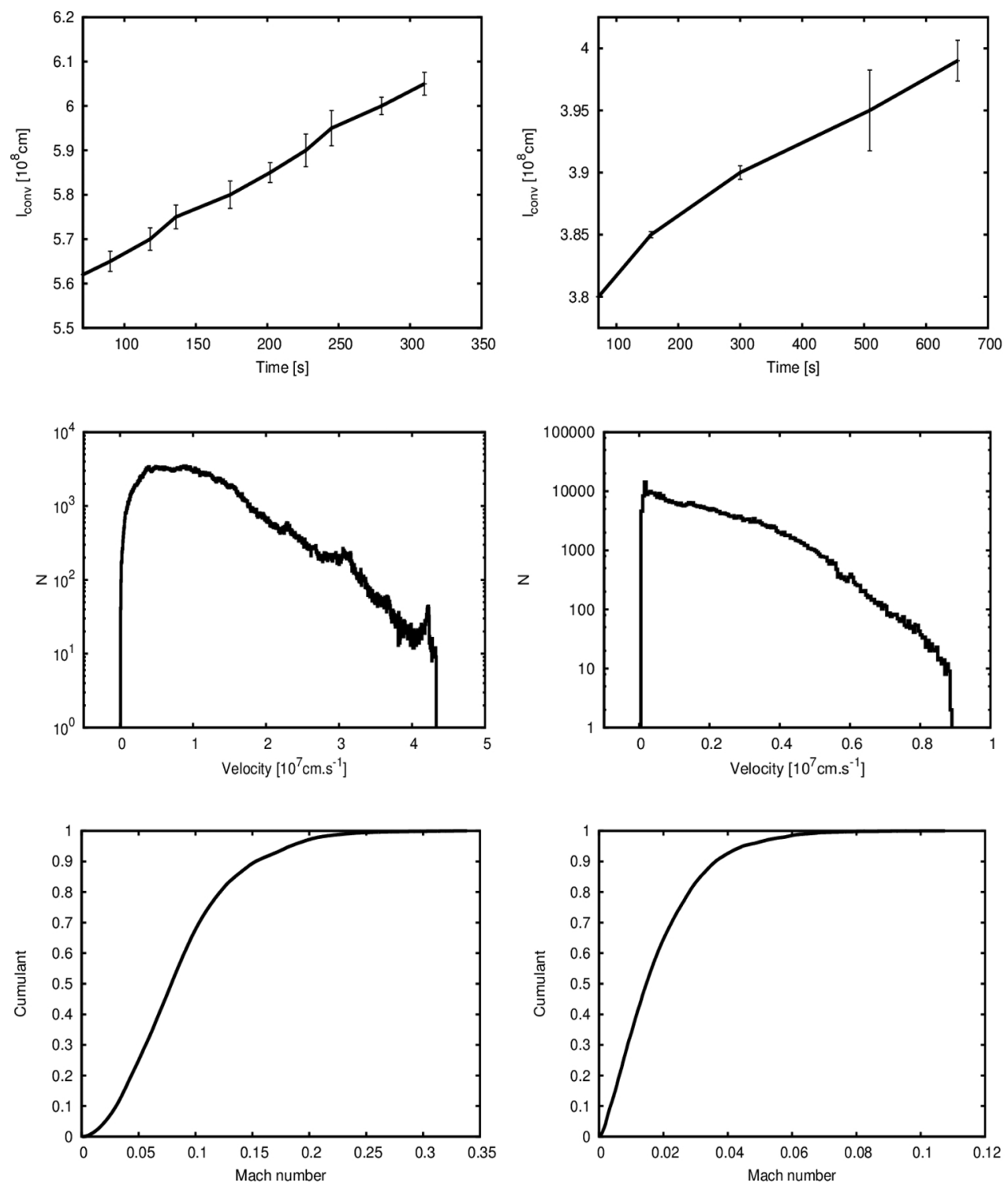

Fig. 2. Propagation of the convective front throughout the accreted envelope as a function of time for models $1.0 M_{\odot} \mathrm{CO}$ (panel $a$ ) and $1.25 M_{\odot} \mathrm{ONe}($ panel $b)$.

Fig. 3. Velocity histograms for models $1.0 M_{\odot} \mathrm{CO}$ at $t=310 \mathrm{~s}$ (panel $a$ ) and $1.25 M_{\odot}$ ONe at $t=657 \mathrm{~s}($ panel $b$ ). The discontinuities in the velocity are due to different stages of the turbulent mixing during the burning advance.

Fig. 4. Cumulant distribution function of the Mach number for models $1.0 M_{\odot} \mathrm{CO}$ at $t=310 \mathrm{~s}$ (panel a) and $1.25 M_{\odot} \mathrm{ONe}$ at $t=657 \mathrm{~s}$ (panel $b$ ). The cumulant distribution shows the probability that the Mach number is equal or below a certain value.

Table 1. Main characteristics of the computed models.

\begin{tabular}{cccccccccc}
\hline \hline Nova & Size $(\mathrm{km} \times \mathrm{km})$ & $M_{\mathrm{wd}}\left(M_{\odot}\right)$ & $\mathrm{g}\left(\mathrm{cm} \mathrm{s}^{-2}\right)$ & $\mathrm{Hp}(\mathrm{km})$ & $d(\mathrm{~km})$ & $t_{\text {inst }}(\mathrm{s})$ & $t_{\mathrm{Hp}}(\mathrm{s})$ & $Z$ & $T_{\text {base }}(\mathrm{K})$ \\
\hline $\mathrm{CO}$ & $800 \times 800$ & 0.8 & $-2.20 \times 10^{8}$ & 156.8 & 627.2 & 60 & 326 & 0.0264 & $1.12 \times 10^{8}$ \\
$\mathrm{CO}$ & $800 \times 800$ & 1.0 & $-4.46 \times 10^{8}$ & 71.2 & 284.6 & 40 & 149 & 0.0295 & $1.10 \times 10^{8}$ \\
$\mathrm{CO}$ & $800 \times 800$ & 1.15 & $-8.32 \times 10^{8}$ & 36.7 & 146.9 & 20 & 66 & 0.0330 & $1.07 \times 10^{8}$ \\
$\mathrm{ONe}$ & $800 \times 400$ & 1.15 & $-8.32 \times 10^{8}$ & 27.6 & 110.5 & 5 & 194 & 0.0530 & $1.01 \times 10^{8}$ \\
$\mathrm{ONe}$ & $800 \times 400$ & 1.25 & $-1.18 \times 10^{9}$ & 16.6 & 66.4 & 4 & 133 & 0.0652 & $1.01 \times 10^{8}$ \\
\hline
\end{tabular}

In Fig. 6, we show the evolution of the metallicity throughout the envelope with time for $\mathrm{CO}$-rich (panel a) and $\mathrm{ONe}$ rich substrates (panel b) until the convective front reaches 4 pressure scale heights. Since the base density and peak temperatures are higher for more massive white dwarfs, the reaction rates increase, which translates into substantial nuclear processing that involves heavier elements. More massive white dwarfs result in a higher metallicity enhancement. For instance, in CO-rich substrates, the final metallicities at the time we stop the simulations are $Z=0.0268$ for $0.8 M_{\odot}, Z=0.0772$ for $1.0 M_{\odot}$, and $Z=0.2651$ for $1.15 M_{\odot}$. In ONe-rich substrates, we obtain $Z=0.0866$ for $1.15 M_{\odot}$, and $Z=0.1174$ for $1.25 M_{\odot}$. These final mean metallicities are higher than those reported in Table 1 because they are measured at the time the convective front almost reached the upper boundary ( $\sim 650 \mathrm{~km}$ above the CEI for CO models, and $\sim 250 \mathrm{~km}$ for ONe models $)^{8}$.

Peak temperatures are determined by the pressure achieved at the base of the envelope before ignition. Accordingly, more massive white dwarfs reach higher pressures and higher peak temperatures; see radial temperature profiles, measured at the

8 Final times are $t=333 \mathrm{~s}$ for model $0.8 M_{\odot} \mathrm{CO}, t=310 \mathrm{~s}$ for model $1.0 M_{\odot} \mathrm{CO}, t=218 \mathrm{~s}$ for model $1.15 M_{\odot} \mathrm{CO}, t=667 \mathrm{~s}$ for model $1.15 M_{\odot} \mathrm{ONe}$, and $t=657 \mathrm{~s}$ for model $1.25 M_{\odot} \mathrm{ONe}$. 

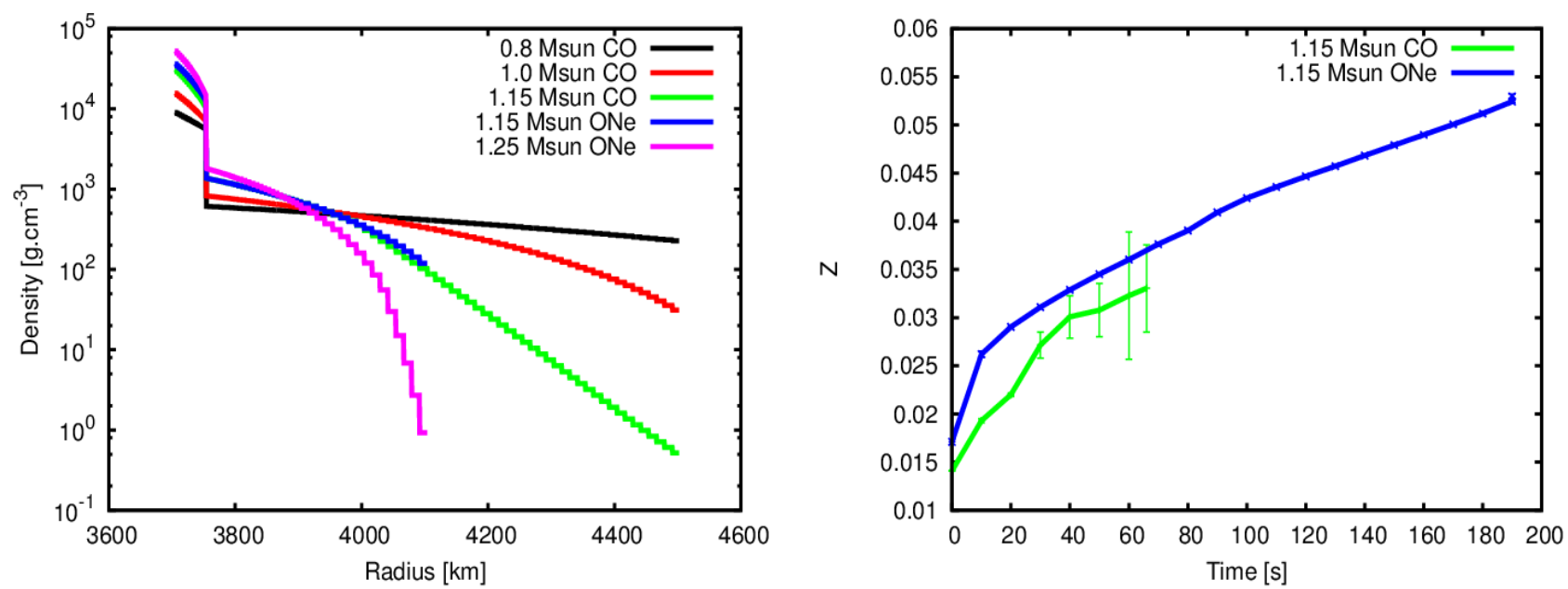

Fig. 5. Panel $a$ : initial density profiles for the five computed models. Panel $b$ : comparison of the time evolution of the mean metallicity throughout the envelope for models $1.15 M_{\odot} \mathrm{CO}$ and $1.15 M_{\odot}$ ONe until the convective front reaches 4 pressure scale heights above the CEI.
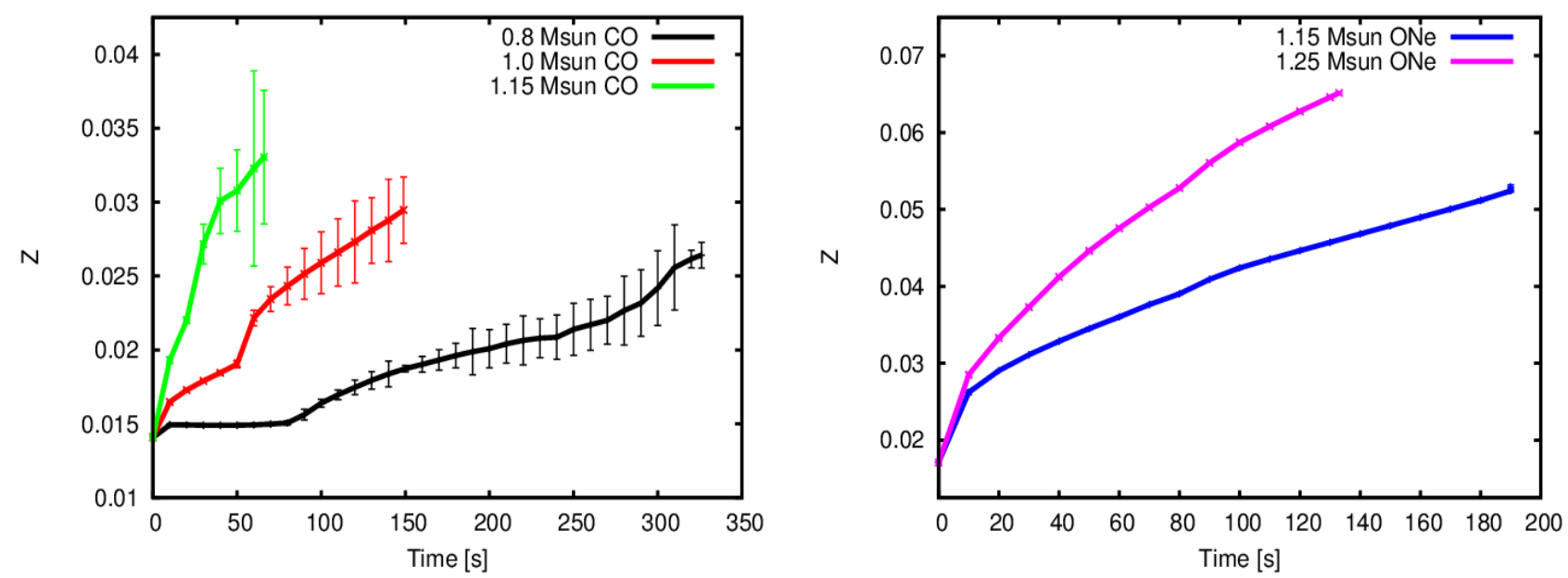

Fig. 6. Evolution of the mean metallicity with time in CO-rich (panel $a$ ) and ONe-rich (panel $b$ ) substrates at 4 pressure scale heights above the CEI, respectively. More massive white dwarfs result in larger metallicity enhancements.
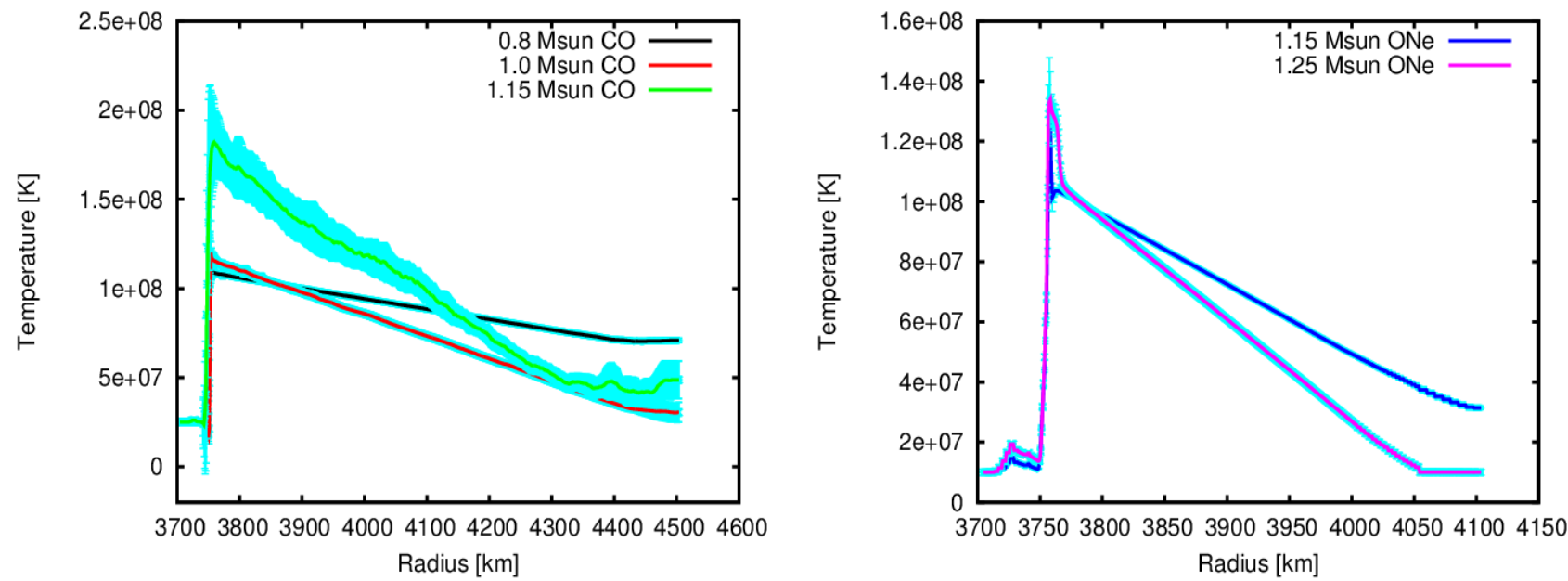

Fig. 7. Averaged radial temperature profile in CO-rich (panel $a$ ) and ONe-rich (panel $b$ ) substrates at the time the convective front almost reaches the upper boundary, $650 \mathrm{~km}$ and $250 \mathrm{~km}$ above the CEI, respectively. Base temperatures are higher in more massive white dwarfs because of higher pressures at the base of the envelope. 

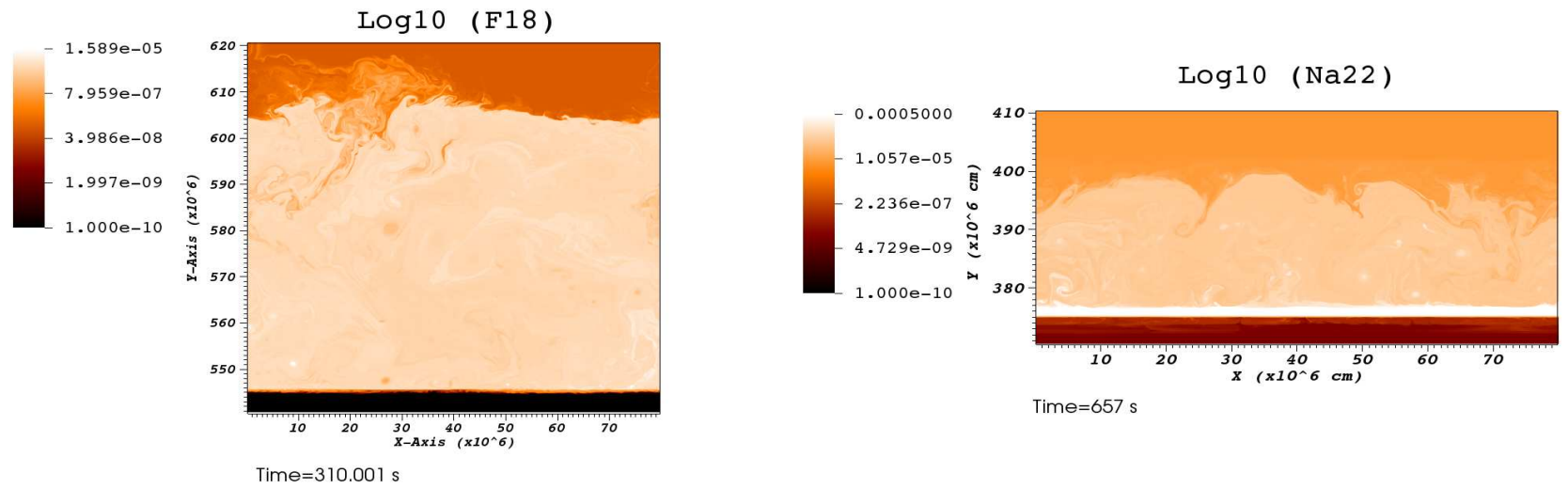

Fig. 8. Development of the initial fluid instabilities at the CEI at $t=310 \mathrm{~s}$, in terms of ${ }^{18} \mathrm{~F}$ mass fraction and in logarithmic scale, for model $1.0 M_{\odot}$ $\mathrm{CO}$ (panel $a$ ), and at $t=657 \mathrm{~s}$, in terms of ${ }^{22} \mathrm{Na}$ mass fraction and in logarithmic scale, for model $1.25 M_{\odot}$ ONe (panel $b$ ). Identical plots are obtained for the other chemical species.

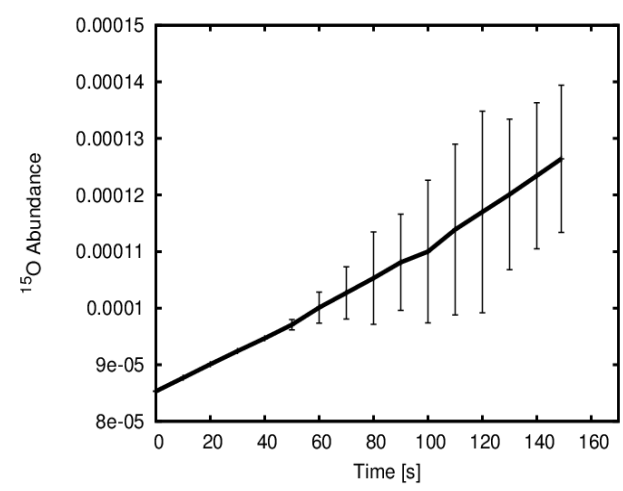

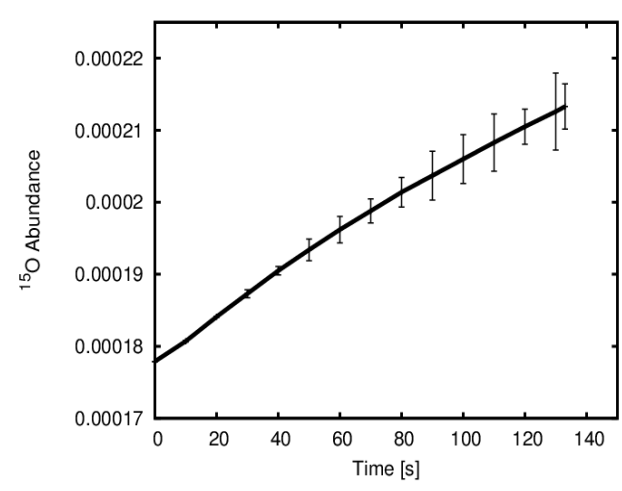

Fig. 9. Evolution of the mean ${ }^{15} \mathrm{O}$ abundance with time throughout the envelope until the convective front reaches 4 pressure scale heights, for models $1.0 M_{\odot} \mathrm{CO}$ (panel $a$ ) and $1.25 M_{\odot} \mathrm{ONe}$ (panel $b)$. time the convective front reached the upper boundary, in panels $a$ and $b$ of Fig. 7. The base temperatures in Fig. 7 are higher than those reported in Table 1 because these are measured at the end of the simulation. At the time we stop the simulations, base temperatures still keep rising, but we expect them to level off when detachment results. The maximum temperatures (the peak value is not yet reached) are lower in ONe-rich substrates at the time we stop the simulations, although we would also expect them to be higher for ONe models than for $\mathrm{CO}$ models at the time of detachment (José \& Hernanz 1998). In ONe-rich substrates, the nuclear activity extends beyond the $\mathrm{CNO}$ mass region, shifting toward the $\mathrm{MgAl}$ and $\mathrm{NeNa}$ regions where heavier isotopes are produced; see the development of fluid instabilities in terms of ${ }^{18} \mathrm{~F}$ and ${ }^{22} \mathrm{Na}$ mass fractions depending on the adopted underlying white dwarf in Fig. 8. In Fig. 9 we show the evolution of the ${ }^{15} \mathrm{O}$ abundance for models $1.0 M_{\odot} \mathrm{CO}$ (panel a) and $1.25 M_{\odot} \mathrm{ONe}$ (panel b). The dispersion found in $\mathrm{ONe}$ models is again smaller than for $\mathrm{CO}$ models, indicating a more uniform enhancement.

\section{Discussion}

In this paper, we have explored the viability of KelvinHelmholtz instabilities operating at the CEI as a mechanism for self-enrichment of the accreted envelope with material from the underlying white dwarf, using various substrates and white dwarf masses. While this was partially investigated by Glasner et al. (2012) in the framework of $\mathrm{CO}$, pure He, pure ${ }^{16} \mathrm{O}$, and pure ${ }^{24} \mathrm{Mg}$ substrates, our work adopts a more realistic composition for an ONe-rich substrate and extends the analysis for a range of white dwarf masses. All the initial models have been self-consistently computed (in 1D), and hence, the adopted envelope masses are also more realistic. The $2 \mathrm{D}$ simulations reported in this paper show that ONe-rich substrates produce a longer dredge-up episode and higher resulting metallicities within the accreted envelope. Finally, we also find more mixing and more energetic explosions for more massive white dwarfs.

A detailed analysis of the development of turbulence in 2D and $3 \mathrm{D}$ frameworks in classical nova explosions and characterization of its intermittent behavior is currently underway. It is worth noting that the convective pattern found in our simulations is an unavoidable consequence of the assumed 2D configuration, forcing the fluid motion to behave differently than in 3D convection (Shore 2007; Meakin \& Arnett 2007). In 2D, the convective cells are forced to merge into large convective eddies with a size comparable to the envelope height. Nevertheless, the levels of metallicity enhancements and the essential explosion properties are recovered in a 2D framework, since fully $3 \mathrm{D}$ simulations differ qualitatively, but not quantitatively, from 2D simulations (Casanova et al. 2011b).

The different envelope size in our 2D simulations, although imposed by the properties of the underlying white dwarf, makes it hard to quantify the final envelope metallicities. The metallicity values reported in this work represent lower limits since the simulations were stopped when the convective front reaches the top of the computational domain and much before the expected detachment of the layers occurs. Extended models are necessary to simulate the entire nova outburst from mixing to the final expansion of the envelope and to quantify rigorously the dredge-up and investigate the progress of the explosion and subsequent ejection. Since our goal is to investigate the mixing 
mechanism during the TNR, we start the simulations from 1D models that assume no premixing. However, forcing the initial 1D model to be in hydrostatic equilibrium after mapping into $2 \mathrm{D}$ imposes a restriction on the portion of the envelope that can be computed, since the density dramatically decreases and produces numerical underflows. A procedure to expand the initial models under hydrostatic equilibrium was explored by Zingale et al. (2002). These authors stopped placing the initial model into hydrostatic equilibrium when a certain density cutoff was reached and added a portion of matter with very low constant density on top of it. This method was found to have a negligible impact on their results. We plan to investigate a similar procedure to extend the initial models in future calculations

A final aspect needs further discussion. In our 1D solar accretion models, the envelope is fully convective when the base temperature is $\sim 9 \times 10^{7} \mathrm{~K}$. A realistic comparison between $\mathrm{CO}$ and ONe models should be made when the rates for proton capture on ${ }^{12} \mathrm{C}$ and ${ }^{20} \mathrm{Ne}$ are similar. At the time we map our models (base temperatures of $10^{8} \mathrm{~K}$ ), the proton capture rate on ${ }^{12} \mathrm{C}$ is much faster than that on ${ }^{20} \mathrm{Ne}$. Therefore, $\mathrm{CO}$ models should be mapped at earlier times to be consistent with $\mathrm{ONe}$ models, regarding the length of the mixing process. Previous studies in 2D (Glasner et al. 1997, 2007) investigated how mapping the initial 1D models at lower temperatures $\left(\sim 7 \times 10^{7} \mathrm{~K}\right)$ influences the overall mixing. However, the authors found minor impact on the results.

Acknowledgements. Part of the software used in this work was developed by the DOE-supposed ASC/Alliances Center for Astrophysical Thermonuclear Flashes at the University of Chicago. This work has been partially supported by the Spanish MINECO grant AYA2017-86274-P, by the E.U. FEDER funds, by the AGAUR/Generalitat de Catalunya grant SGR-661/2017, and by the US Department of Energy, Office of Science, Office of Nuclear Physics. We also acknowledge the Barcelona Supercomputing Center for a generous allocation of time at the MareNostrum supercomputer. This article benefited from discussions within the ChETEC COST Action (CA16117).

\section{References}

Andreä, J., Dreschel, H., \& Starrfield, S. 1994, A\&A, 291, 869

Casanova, J., José, J., García-Berro, E., Calder, A., \& Shore, S. N. 2010, A\&A, 513, L5

Casanova, J., José, J., García-Berro, E., Calder, A., \& Shore, S. N. 2011a, A\&A, 527, A5

Casanova, J., José, J., García-Berro, E., Calder, A., \& Shore, S. N. 2011b, Nature, 478,490

Casanova, J., José, J., García-Berro, E., \& Shore, S. N. 2016, A\&A, 595, A28

Colella, P., \& Woodward, P. R. 1984, J. Comput. Phys., 54, 174

Downen, L. N., Iliadis, C., José, J., \& Starrfield, S. 2013, ApJ, 762, 105

Durisen, R. H. 1977, ApJ, 213, 145

Fryxell, B., Olson, K., Ricker, P., et al. 2000, ApJ, 399, 646
Fujimoto, M. Y. 1988, A\&A, 198, 163

Fujimoto, M., \& Iben, I., Jr. 1992, ApJ, 399, 646

Gehrz, R. D., Truran, J. W., Williams, R. E., \& Starrfield, S. 1998, PASP, 110,3

Glasner, S. A., Livne, E., \& Truran, J. W. 1997, ApJ, 475, 754

Glasner, S. A., Livne, E., \& Truran, J. W. 2007, ApJ, 665, 1321

Glasner, S. A., Livne, E., \& Truran, J. W. 2012, MNRAS, 427, 2411

Glasner, S. A.Livne, E., \& Truran, J. W. 2014, in Stella Novae: Past and Future Decades, eds. P. A. Woudt, \& V. A. R. M. Ribeiro, ASP Conf. Ser., 490, 295 Iben, I., Jr., Fujimoto, M. Y., \& MacDonald, J. 1991, ApJ, 375, L27

Iben, I., Jr., Fujimoto, M. Y., \& MacDonald, J. 1992, ApJ, 388, 521

Iliadis, C., Longland, R., Champagne, A. E., Coc, A., \& Fitzgerald, R. 2010, Nucl. Phys. A, 841, 31

José, J. 2016, in Stellar Explosions: Hydrodynamics and Nucleosynthesis (Boca Raton: CRC/Taylor and Francis)

José, J., \& Hernanz, M. 1998, ApJ, 494, 680

Jose, J., \& Shore, N. S. 2008, in Classical Novae, 2nd edn., eds. M. F. Bode, \& A. Evans (Cambridge: Cambridge Univ. Press), 77

José, J., Halabi, G. M., \& El Eid, M. F. 2016, A\&A, 593, A54

Kelly, K. J., Iliadis, C., Downen, L., José, J., \& Champagne, A. 2013, ApJ, 777, $130 \mathrm{~K}$

Kippenhahn, R., \& Thomas, H. 1978, A\&A, 63, 265

Kovetz, A., \& Prialnik, D. 1985, ApJ, 291, 812

Kovetz, A., \& Prialnik, D. 1997, ApJ, 477, 356

Kutter, G. S., \& Sparks, W. M. 1987, ApJ, 321, 386

Kutter, G. S., \& Sparks, W. M. 1989, ApJ, 340, 985

Landau, L. D., \& Lifshitz, E. M. 1987, in Fluid Mechanics, 2nd edn. (Butterworth-Heinemann)

Lesieur, M. 2008, in Turbulence in Fluids, New Trends in Turbulence (Springer) Livio, M., \& Truran, J. W. 1987, ApJ, 318, 316

Livio, M., \& Truran, J. W. 1990, Proc. 5th Florida Workshop in Nonlinear Astronomy, 617, 126

MacDonald, J. 1983, ApJ, 273, 289

Meakin, C. A., \& Arnett, D. 2007, ApJ, 667, 448

Pope, S. B. 2000, in Turbulent Flows (Cambridge: Cambridge Univ. Press)

Prialnik, D., \& Kovetz, A. 1984, ApJ, 281, 367

Ritossa, C., García-Berro, E., \& Iben, I., Jr. 1996, ApJ, 460, 489

Shara, M. M. 1982, ApJ, 261, 649

Shen, K., \& Bildsten, L. 2009, ApJ, 281, 367

Shore, S. N. 2007, in Astrophysical Hydrodynamics: An Introduction (Darmstadt: Wiley/VHC)

Shore, S. N., Livio, M., \& van den Heuval, E. P. J. 1994, in Interacting binaries. Saas-Fee Advanced Course 22: Interacting Binaries (Berlin: Springer)

Snijders, M. A. J., Batt, T. J., Roche, P. F., et al. 1987, MNRAS, 228, 329

Sparks, W. M., \& Kutter, G. S. 1987, ApJ, 321, 394

Spitzer, L. 1962, in Physics of Fully Ionized Gases (New York: Wiley/Interscience)

Starrfield, S., Truran, J. W., Wiescher, M., \& Sparks, W. 1998, MNRAS, 296 , 502

Starrfield, S., Iliadis, C., \& Hix, W. R. 2008, in Classical Novae, 2nd edn., eds. M. F. Bode, \& A. Evans (Cambridge: Cambridge Univ. Press), 77

Starrfield, S., Iliadis, C., \& Hix, R. W. 2016, PASP, 28, 963

Timmes, F. X. 2000, ApJ, 528, 913

Timmes, F. X., \& Arnett, D. 2000, ApJS, 125, 277

Timmes, F. X., \& Swesty, F. D. 2000, ApJS, 126, 501

Vanlandingham, K., Starrfield, S., \& Shore, S. N. 1997, MNRAS, 290, 87

van Leer, B. 1979, J. Comput. Phys., 32, 101

Yaron, O., Prialnik, D., Shara, M. M., \& Kovetz, A. 2005, ApJ, 623, 398

Zingale, M., Dursi, L. J., Zuhone, J., et al. 2002, ApJS, 143, 539 